\title{
Salivary Alkaline Phosphatase Level in People with Healthy Periodontium and Generalised Chronic Periodontitis
}

\author{
Dr. Pramod Kumar Koirala, ${ }^{1}$ Dr. Shaili Pradhan, ${ }^{1}$ Dr. Ranjita Shrestha Gorkhali ${ }^{1}$ \\ 'Periodontology and Oral Implantology Unit, Department of Dental Surgery, \\ National Academy of Medical Sciences, Bir Hospital, Kathmandu, Nepal.
}

\begin{abstract}
Introduction: Periodontal disease is a multifactorial chronic inflammatory disease affecting the soft and hard supporting structures of teeth leading to alveolar bone loss and exfoliation of involved teeth when left untreated. Traditional periodontal diagnostic methods are not always able to assess present disease states. Saliva as possible biological sample helps measure current disease state, monitor treatment, and indicate prognosis. Oral fluids contain mediators of disease that include microbial host response and bone-specific resorptive markers.

Objective: To investigate and compare salivary alkaline phosphatase level in people with healthy periodontium and generalised chronic periodontitis.

Methods: This cross-sectional analytical study was conducted in the department of dental surgery, Bir hospital from January to December 2017. Ethical clearance was obtained from institutional review board. Unstimulated saliva sample for the evaluation of alkaline phosphatase was collected from 35 generalised chronic periodontitis (GCP) patients and 35 volunteers with healthy periodontium after informed consent was obtained and periodontal parameters were recorded. Data collected was analysed using SPSS software v.16.

Results: Three and half fold increased level of salivary alkaline phosphatase level in GCP patients than healthy volunteers was found which is highly significant. Correlation of salivary alkaline phosphatase level with clinical features of the chronic periodontal disease was observed.

Conclusion: An expected benefit of an oral fluid-based periodontal diagnostics would be identification of highly susceptible individuals prior to aggressive disease. Timely detection and diagnosis of disease offer earlier, less invasive, and more cost-effective treatment.

Keywords: Alkaline phosphatase; biomarker; inflammation; periodontitis; saliva.
\end{abstract}

\section{INTRODUCTION}

Chronic periodontitis is a disease of multifactorial etiology with unpredictable progression, resulting in inflammation and destruction of the supporting structures. ${ }^{1}$ Alkaline phosphatase (ALP) is an enzyme that hydrolyses organic phosphate ester linkages which plays key roles in bone homeostasis. ALP is released from neutrophils during inflammation, from osteoblasts during bone formation, and periodontal fibroblasts during periodontal regeneration showing dual involvement of periodontal inflammation and regeneration. ${ }^{2}$

\section{Correspondence:}

Dr. Pramod Kumar Koirala

Periodontology and Oral Implantology Unit, Department of Dental Surgery, National Academy of Medical Sciences, Bir Hospital, Kathmandu, Nepal.

email:drpkkoirala@gmail.com

\section{Citation}

Koirala PK, Pradhan S, Gorkhali RS. Salivary Alkaline Phosphatase Level in People with Healthy Periodontium and Generalised Chronic Periodontitis. J Nepal Soc Perio Oral Implantol. 2020 JulDec;4(8):68-72.
The existing clinical methods of periodontal diagnosis provide information about disease presence after its onset. More reliable diagnostic investigations are being developed based on identification and estimation of various mediators involved in immunological or host response events to elucidate disease initiation, progression, or state of remission. ${ }^{3}$ Patients at risk for active progressive periodontal disease needs improved methods of periodontal diagnosis. ${ }^{4}$ ALP may serve as a marker in periodontal treatment planning and monitoring. ${ }^{5}$ Clinically and commercially collection of saliva is safe, non-invasive, simple, and can be repeated with minimum discomfort. ${ }^{6}$

According to World Health Organisation, severe periodontitis leading to tooth loss was found in 5-15\% populations worldwide. ${ }^{7}$ Periodontal problems occupy the second place among oral health problems in Nepal. ${ }^{8}$ This study investigated saliva ALP levels in generalised chronic periodontitis cases comparing with people having healthy periodontium.

\section{METHODS}

This cross-sectional analytical study conducted between January and December of 2017 in the Department of Dental 
Surgery, Bir Hospital included 35 healthy individuals without periodontitis and 35 patients with generalised chronic periodontitis (GCP) after sample size calculation. Patients having at least 20 teeth were included. Those suffering from chronic systemic inflammatory and infectious conditions, pregnant and lactating mothers, post menopausal females, those undergoing any periodontal therapy with antibiotic intake in the past three months, and current smokers were excluded.

The participants had to undergo periodontal examination by manual circumferential probing using millimeter scale University of North Carolina 15 (UNC15R, Hu-Friedy, Chicago, IL, USA) manual periodontal probe. Full mouth periodontal charting was done. Clinical periodontal parameters which are related to tissue destruction: plaque index, ${ }^{9}$ gingival index, ${ }^{10}$ periodontal pocket depth (PPD), clinical attachment level (CAL), and gingival bleeding on probing (BOP) were investigated.

A modified method of saliva collection was employed. ${ }^{11}$ The patient was instructed not to eat or drink anything two hours before the collection of saliva. The patient was asked to try and control any facial movements especially those of the mouth. Initially, the saliva already present in the oral cavity was swallowed and unstimulated saliva was collected in the tube by asking the subject to lean forward with a slightly opened mouth (Figure 1). Any remaining saliva in the floor of the mouth was added to the tube after the end of the fiveminute collection period. The various equipment and reagent used for the evaluation of ALP level of saliva sample are shown in Figure 2, 3, 4, and 5.

Level of ALP was estimated with an auto analyser (ERBA Mannheim, Figure 2) by using International Federation of Clinical Chemistry (IFCC) method. For analysis each saliva

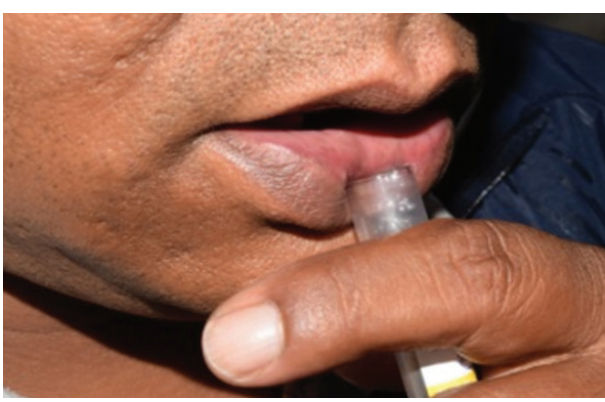

Figure 1: Collection of saliva sample.

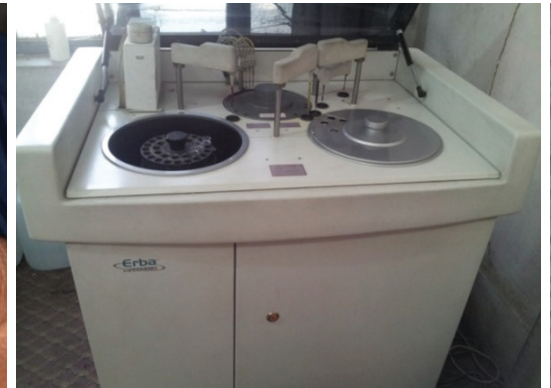

Figure 2: ERBA Mannheim auto analyser. sample was centrifuged at 5000 revolutions per minute (rpm) for 5-10 minutes (Figure 5).

Reagents (Figure 5) were added to about 20 micro liter of supernatant and ALP estimated in U/L. ${ }^{12}$ The hydrolysis of p-Nitrophenyl phosphate to yellow coloured p-Nitrophenol and phosphate is a reaction catalysed by alkaline phosphatase at $\mathrm{pH}$ 10.3. Autoanalyser measured the change in absorbance at $405 \mathrm{~nm}$ wavelength which is proportional to ALP activity.13

Patients were assessed as per the requisite on the basis of objectives of determining and comparing the salivary ALP level in people with healthy periodontium and generalised chronic periodontitis with respect to periodontal parameters.

Data were analysed using the statistical package for the Social Sciences (SPSS) Statistics for Windows, version 16.0 (SPSS Inc., Chicago, Ill., USA). Normally distributed variables are presented as means and standard deviations and quantitative variables as frequencies. Independent t-test was used to compare the enzyme levels in saliva. Pearson correlation coefficient between periodontal parameters and biochemical variables were calculated. Significance was determined at $5 \%$ $(\mathrm{P}<0.05)$.

\section{RESULTS}

A total of 70 individuals (minimum 35 years, maximum 54 years) participated in the study. Among them, 35 generalised chronic periodontitis patients (test group) with mean age of $43.29 \pm 6.08$ years and 35 periodontally healthy volunteers (control group) with mean age of $39.2 \pm 5.87$ years (Table1) underwent measurement of all clinical parameters along with saliva at initial visit. The obtained results were tabulated (Table 2, 3, and 4) and presented in diagrams (Figure 6).
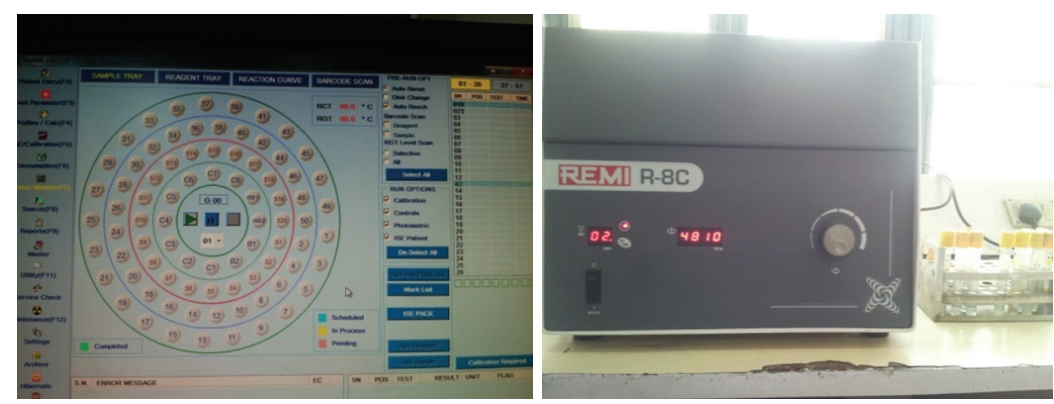

Figure 4: Auto analyser computer display. Figure 5: Centrifuge machine and sample. 
Table 1: Distribution of study population by gender and age.

\begin{tabular}{|l|c|c|}
\hline & Healthy subjects & Generalised chronic periodontitis \\
\hline Frequency (n) & 35 & 35 \\
\hline Male $\mathrm{n}(\%)$ & $19(54)$ & $21(60)$ \\
\hline Female $\mathrm{n}(\%)$ & $16(46)$ & $14(45)$ \\
\hline Age $($ Mean \pm SD) & $39.2 \pm 5.87$ & $43.29 \pm 6.08$ \\
\hline
\end{tabular}

Table 2: Periodontal findings in the different groups of study population.

\begin{tabular}{|l|c|c|}
\multicolumn{1}{c|}{ Variables } & $\begin{array}{c}\text { Generalised chronic periodontitis } \\
\text { Mean } \pm \text { SD }\end{array}$ & $\begin{array}{c}\text { Generalised chronic periodontitis } \\
\text { Mean } \pm \text { SD }\end{array}$ \\
\hline Plaque index & $1.95 \pm 0.30$ & $0.45 \pm 0.17$ \\
\hline Gingival index & $1.87 \pm 0.24$ & $0.36 \pm 0.25$ \\
\hline Periodontal pocket depth & $5.28 \pm 0.621$ & $1.78 \pm 0.28$ \\
\hline Clinical attachment level & $5.2 \pm 0.67$ & $1.94 \pm 0.10$ \\
\hline
\end{tabular}

Table 3: Salivary alkaline phosphatase level among the two groups of study population.

\begin{tabular}{|l|c|c|} 
& ALP U/L (Mean \pm SD) & F value \\
\hline Healthy periodontium & $9.65 \pm 6.82$ & 28.32 \\
\hline Generalised chronic periodontitis & $36.94 \pm 29.55$ & $<0.001$ \\
\hline Unpaired t-test to compare the salivary ALP level of periodontitis and healthy cases. \\
\hline
\end{tabular}

Table 4: Correlation between salivary ALP level and gingival index in overall data.

\begin{tabular}{|l|c|c|cc|}
\hline \multicolumn{1}{|c|}{ Coefficient } & Estimate & Standard error & T value & P value \\
\hline Intercept & 4.74 & 4.55 & 1.04 & $<0.001$ \\
\hline Gingival index & 16.59 & 3.34 & 4.97 & \\
\hline
\end{tabular}

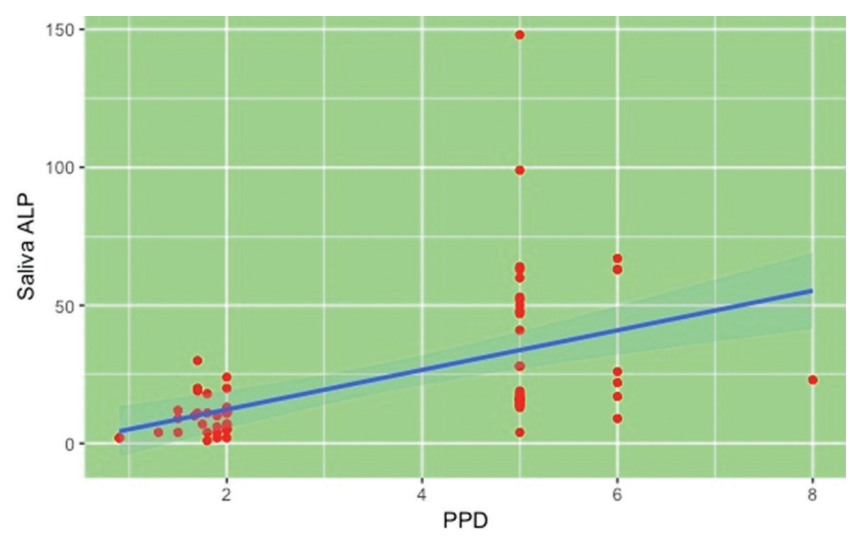

Figure 6: Periodontal pocket depth plotted against salivary alkaline phosphatase.

iAs presented in Table 4, the gingival index was found to have positive correlation which was statistically significant $(\mathrm{P}$ value $<0.001$ ) to the level of salivary ALP. From the analysis of covariates (ANOVA) it was observed that when GI value increases by 1 , there will be 16.59 unit increase in salivary ALP level.

The graphical representationof the level of ALP in different severity of pocket depth (Figure 6) and clinical attachment level (Figure 7) show that there is positive relationship

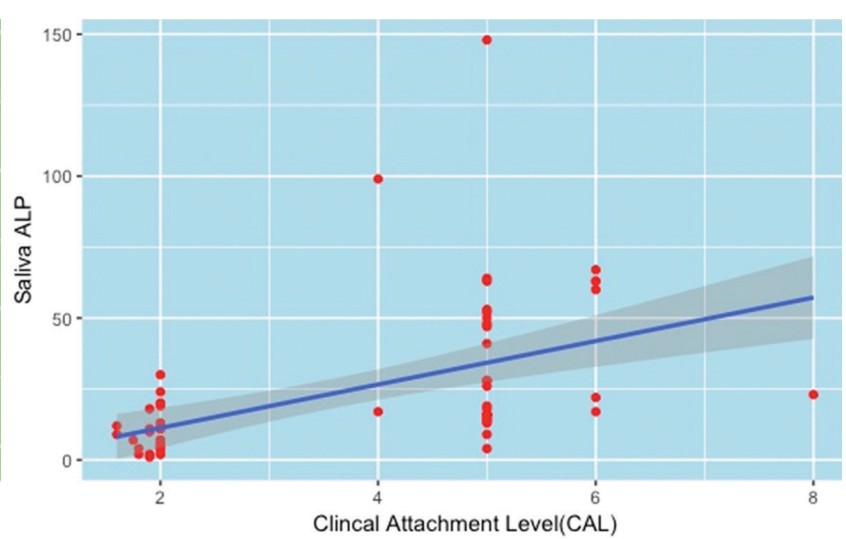

Figure 7: Clinical attachment level plotted against salivary alkaline phosphatase level.

between the variables. These periodontal parameters are directly proportional to ALP level.

\section{DISCUSSION}

People with healthy periodontium with clinical attachment loss $\leq 2 \mathrm{~mm}$ and GCP patients with clinical attachment level $\geq$ $3 \mathrm{~mm}$ and periodontal pocket depth 4-7 $\mathrm{mm}$ in more than $30 \%$ of sites were included. In this study we observed that salivary ALP level was higher in generalised chronic periodontitis patients than people with healthy periodontium. ALP level is 
significantly elevated in generalised chronic periodontitis.

We have compared equal number of objects. These findings are in agreement with previous data. In our study, we found that indicators of both current and prolonged chronic periodontitis status (PPD and CAL respectively) were positively associated with salivary alkaline phosphatase levels in generalised chronic periodontitis patient.

In the current study, it was observed that salivary alkaline phosphatase level in generalised chronic periodontitis is $36.94 \pm 29.55 \mathrm{IU} / \mathrm{L}$ which is three and half times higher than that of healthy people with ALP level of $9.65 \pm 6.58 \mathrm{IU} / \mathrm{L}$ (Table 3). ALP has been shown to be elevated in numerous tissue pathologies of liver, bone, gut and other soft tissues.Since systemic diseases can cause variations in ALP levels, people with such underlying conditions are excluded from the study.

Gingival index and plaque index were found statistically significant in relations to the level of salivary ALP (P value $<0.001$ ). From the analysis of covariates (ANOVA), the test value ( $\mathrm{t}$ value) was significantly high with the change of GI score by 1 , there will be 16.59 unit increase in Saliva ALP level. The analysis has found similar correlations with plaque index. If such relationship could be established in longitudinal studies, it can be a valuable predictor.

Demographic data have shown that family history, gum bleeding, brushing frequencies, and poor oral hygiene was related in the development of generalised chronic periodontitis. The GCP patients are poor in cleaning habits and hygiene and have higher family predispositions of periodontal disease, are of the older age group and have spacing and increased mobility of teeth as well as high rate of gingival bleeding.

This study showed that the mean salivary alkaline phosphatase levels vary with the severity of periodontal disease as subjects with chronic periodontitis had the highest level and those with healthy periodontium had the least. The results suggest that salivary alkaline phosphatase levels increase in periodontal disease. The increase in salivary alkaline phosphatase levels could be traced to increased activities of polymorphonuclear neutrophils, osteoblasts and fibroblasts within the periodontal pocket as a result of persistent inflammation. The increased salivary ALP in periodontitis could also be associated with alveolar bone loss, with a small contribution from the serum. ${ }^{14,15}$

Early investigations of ALP and periodontal disease in an experimental gingivitis model showed a significant correlation between ALP and pocket depth and between ALP and inflammation. ${ }^{16}$ Nakamura and Slots studied a total of 76 enzyme activities in mixed whole saliva and noted higher enzyme activity in individuals with periodontal disease than in non-diseased individuals. ${ }^{17}$ Totan et al. investigated the influence of periodontal disease on ALP. ${ }^{18}$ Salivary samples from patients with confirmed periodontal disease were analysed and revealed that periodontal destruction by measurement of probing depth, gingival bleeding, and suppuration were related to higher ALP levels in saliva. Supporting these results are the findings by Todorovic et al. that increased activity of salivary ALP is seen in patients with periodontal disease in relation to a nondisease control group. ${ }^{19}$

This group further showed a positive correlation between the salivary enzyme activity and gingival index values. As a predictive indicator for future periodontal breakdown, ALP has not been supported by research findings and therefore may best serve as a marker in periodontal treatment planning and monitoring. ${ }^{20,21}$ Trivedi et al. ${ }^{22}$ estimated the ALP level along with other enzymes in unstimulated saliva for the diagnosis of periodontitis in patients with chronic periodontitis and healthy individuals. The results of the study signified increased activities of ALP and other enzymes in unstimulated saliva of patients with chronic periodontitis.

Ramesh et al. estimated the salivary ALP levels in postmenopausal women with and without chronic periodontitis and the results showed a significant increase in salivary ALP levels. ${ }^{23}$

Some researchers have used GCF and others utilised saliva (stimulated/ unstimulated) in the early diagnosis of periodontitis either by cross-sectional or longitudinal studies.

Previous studies have shown that constituents present in saliva can provide important complimentary diagnostic information. Salivaomics Knowledge Base has promoted diagnostic potentials of salivary biomarkers. ${ }^{24}$

There has been some considerable interest in promoting the dental clinic as a venue for general medical diagnosis through physiological measurements (such as weight, height, and blood pressure), finger stick blood tests, and salivary diagnostics. ${ }^{25}$

Development of a practical framework for clinical management of periodontitis using salivary biomarkers will provide a paradigm for exploring the application of general medical diagnostic procedures using saliva in the dental clinic.

Hence we should make habit of requesting our patient to undergo hematological, biochemical or immunological/ microbiological investigations where feasible. This can help both patient and operator in incidental findings of other predisposing factors of periodontal disease and systemic diseases. This will help in future research purposes. The common investigations are cost-effective too. The method of evaluation of spectrophotometry has been made simplified by advances in equipment to complete auto analyser. Highly sensitive chemiluminescence techniques are also available. In addition interventional studies are also necessary to examine the potential benefits of periodontal treatment. ${ }^{26}$ If the consequences of treatment had been included in the study, it would have been more effective. Further longitudinal studies can be recommended. 


\section{CONCLUSION}

Salivary ALP can be considered as a marker of chronic periodontitis as its level is significantly (three and a half times) elevated above that of periodontally healthy people. In majority of cases, the elevation of level of ALP saliva is directly proportional to the level of periodontal parameters.

Further longitudinal studies with larger sample are warranted to confirm our results. Multiple enzymes can be taken as biomarkers in further studies. If access to a portable analyser was available, chair-side diagnosis could have been performed. Cases with undiagnosed systemic disease can be misleading due to the raised level of ALP due to other than periodontal sources like liver, gut, renal pathologies.

\section{Conflict of Interest: None.}

\section{REFERENCES}

1. Page RC, Offenbacher S, Schroeder HE, Seymour GJ, Kornman KS. Advances in the pathogenesis of periodontitis: summary of developments, clinical implications and future directions. Periodontol 2000. 1997;14(1):216-48.

2. Socransky S, Haffajee A, Goodson J, Lindhe J. New concepts of destructive periodontal disease. J Clin Periodontol. 1984;11(1):21-32.

3. Gul SS, Douglas CW, Griffiths GS, Rawlinson A. A pilot study of active enzyme levels in gingival crevicular fluid of patients with chronic periodontal disease. J Clin Periodontol. 2016;43(8):629-36.

4. Socransky SS, Haffajee AD. The bacterial etiology of destructive periodontal disease: current concepts. J Periodontol. 1992;63:322-31.

5. Newman MG, Takei H, Klokkevold PR, Carranza FA, editors. Carranza's clinical periodontology - A south asia edition. 11th ed. New Delhi, India: Saunders, Elsevier; 2011.

6. Moles MG, Dorrego MV. Tissue destruction in periodontitis: bacteria or cytokines fault. Quintessence Int (Berlin, Germany: 1985). 2005;36:299306.

7. Armitage GC. Analysis of gingival crevice fluid and risk of progression of periodontitis. Periodontol 2000. 2004;34(1):109-19.

8. Malla S, Shrestha R, Dhami B, Gupta S, Deo S. Knowledge and practices of periodontal health and oral hygiene among BDS students. J Nepal Soc Periodontol Oral Implantol. 2017;1(2):51-4.

9. Silness J, Löe H. Periodontal disease in pregnancy II. Correlation between oral hygiene and periodontal condition. Acta Odontol Scand. 1964;22(1):121-35.

10. Löe H, Silness J. Periodontal disease in pregnancy I. Prevalence and severity. Acta Odontol Scand. 1963;21(6):533-51.

11. Navazesh M, Christensen C, Brightman V. Clinical criteria for the diagnosis of salivary gland hypofunction. J Dent Res. 1992;71(7):1363-9.

12. Navazesh M, Christensen C. A comparison of whole mouth resting and stimulated salivary measurement procedures. J Dent Res. 1982;61(10):1158-62.

13. Luke R, Khan SN, Iqbal PS, Soman RR, Chakkarayan J, Krishnan V. Estimation of specific salivary enzymatic biomarkers in individuals with gingivitis and chronic periodontitis: A clinical and biochemical study. J Int Oral Health. 2015;7(9):54.

14. Chapple I, Matthews J, Thorpe G, Glenwright H, Smith J, Saxby M. A new ultrasensitive chemiluminescent assay for the site-specific quantification of alkaline phosphatase in gingival crevicular fluid. J Periodontal Res. 1993;28(4):266-73.

15. Todorovic T, Dozic I, Barrero MV, Ljuskovic B, Pejovic J, Marjanovic M, et al. Salivary enzymes and periodontal disease. Medicina Oral Patología Oral Cirugía Bucal Ed Inglesa. 2006;11(2):4.

16. Ishikawa I, Cimasoni G. Alkaline phosphatase in human gingival fluid and its relation to periodontitis. Arch Oral Biol. 1970;15(12):1401-4.

17. Nakamura M, Slots J. Salivary enzymes: origin and relationship to periodontal disease. J Periodontal Res. 1983;18(6):559-69.

18. Totan A, Greabu M, Totan C, Spinu T. Salivary aspartate aminotransferase, alanine aminotransferase and alkaline phosphatase: possible markers in periodontal diseases? Clin Chemi Lab Med (CCLM). 2006;44(5):612-5.

19. Todorović T, Doćžć I, Pavlica D, Marković D, Ivanović M, Brajović G, et al. Use of saliva as a diagnostic fluid in dentistry. Srpski arhiv za celokupno lekarstvo. 2005;133(7-8):372-8.

20. McCauley LK, Nohutcu RM. Mediators of periodontal osseous destruction and remodeling: principles and implications for diagnosis and therapy. J Periodontol. 2002;73(11):1377-91.

21. Nakashima K, Giannopoulou C, Andersen E, Roehrich N, Brochut P, Dubrez B, et al. A longitudinal study of various crevicular fluid components as markers of periodontal disease activity. J Clin Periodontol. 1996;23(9):832-8.

22. Trivedi S, Lal N, Mahdi AA, Singh B, Pandey S. Association of salivary lipid peroxidation levels, antioxidant enzymes, and chronic periodontitis. Int J Perio Resto Dent. 2015;35(2).

23. Ramesh A, Bhandary R, Thomas B, D'Souza SR, Kumari S. Alkaline phosphatase-a diagnostic marker of periodontitis in postmenopausal women-a biochemical study. Nitte Univ J Health Sci. 2013;3(4):71.

24. Baum B, Yates III J, Srivastava S, Wong D, Melvin J. Scientific frontiers: emerging technologies for salivary diagnostics. Adv Dent Res. 2011;23(4):360-8.

25. Urdea M, Neuwald P, Greenberg B, Glick M, Galloway J, Williams D, et al. Saliva, diagnostics, and dentistry. Adv Dent Res. 2011;23(4):353-9.

26. Pradhan S, Koirala P. Alkaline phosphatase levels before and after nonsurgical periodontal therapy. J Nepal Soc Perio Oral Implantol 2019;3(6):62-5. 